\title{
Colored black holes and Kac-Moody algebra
}

\author{
Gaston Giribet@ and Luciano Montecchio \\ Physics Department, University of Buenos Aires FCEyN-UBA and IFIBA-CONICET Ciudad \\ Universitaria, pabellón 1, 1428 Buenos Aires, Argentina
}

(Received 24 November 2021; accepted 22 February 2022; published 4 March 2022)

\begin{abstract}
We demonstrate that the near horizon symmetries of black holes in Einstein-Yang-Mills (EYM) theory are generated by an infinite-dimensional algebra that contains, in addition to supertranslations and superrotations, a non-Abelian loop algebra. This means that the Virasoro-Kac-Moody structure of EYM in asymptotically flat spacetimes has an exact analog in the near horizon region.
\end{abstract}

DOI: 10.1103/PhysRevD.105.064006

\section{INTRODUCTION}

In 2015, Hawking conjectured that, in their near horizon limit, black holes might exhibit an infinite-dimensional symmetry [1] similar to the supertranslations that appear near null infinity in asymptotically flat spacetimes [2-4]. His conjecture was motivated by the recent developments connecting the subjects of asymptotic symmetries, soft theorems, and memory effect in gravity and gauge theories [5]. The original idea was that horizon supertranslations could have something to do with the black hole information puzzle [6], an interesting idea that gave rise to a thoughtful debate [7-9]. Nevertheless, regardless whether relevant or not for the information loss problem specifically, the emergence of infinite-dimensional symmetries near the black hole event horizons turned out to be an interesting discovery on its own right, as it suggests that there might still be important lessons to be learnt from symmetries about the infrared structure of gravity and gauge theories.

The existence of infinite-dimensional symmetries in the vicinity of black hole horizons was made precise in [10], where it was shown that, in addition to supertranslations, black holes also exhibit superrotations in their proximity. Further details of these symmetries and their associated charges were given in $[11,12]$, and in references thereof. The study of infinite-dimensional symmetries in the near horizon region has antecedents [13-15], and more recently it led to interesting developments and generalizations; see for instance [16-25].

It was shown in [16] that the addition of Abelian gauge fields results in a further enhancement of the near horizon symmetry, yielding a new set of supertranslation currents

Published by the American Physical Society under the terms of the Creative Commons Attribution 4.0 International license. Further distribution of this work must maintain attribution to the author(s) and the published article's title, journal citation, and DOI. Funded by SCOAP. for each $U(1)$ commuting factor in the gauge group, at least. Here, we will consider the case of Einstein gravity coupled to Yang-Mills theory for an arbitrary gauge group $G$, and we will show that, in addition to supertranslations and superrotations, the black holes of the theory exhibit an infinite-dimensional symmetry that is generated by a nonAbelian loop algebra. This means that, as it happens in Einstein-Yang-Mills theory in asymptotically flat spacetimes [26], a Virasoro-Kac-Moody structure emerges in the near horizon region of colored black holes. In other words, the same symmetry enhancement phenomenon of the nonAbelian algebra discovered by Barnich and Lambert at null infinity also occurs near the black hole event horizon. Actually, the full algebra we will encounter in the near horizon limit differs from the one found near null infinity, the difference being the structure constants that connect supertranslations to superrotations, cf. [16]; however, the Virasoro-Kac-Moody piece matches exactly.

The paper will be organized as follows: In Sec. II we will consider the near horizon symmetries in Einstein-YangMills theory. We will present a sensible set of boundary conditions at the horizon that, on the one hand, permit to accommodate the physically relevant solutions such as colored black holes and, on the other hand, turn out to be preserved by an infinite set of diffeomorphisms and gauge transformations. These boundary conditions are the generalization of those proposed in [10] to the non-Abelian case. In Sec. III we will study the algebra of diffeomorphisms and gauge transformations preserving the prescribed boundary conditions, and we will show that it turns out to be an infinite-dimensional algebra that contains a Kac-Moody subalgebra. Section IV contains a brief discussion about the relevance of our result.

\section{NON-ABELIAN HORIZONS}

Let us consider a four-dimensional spacetime $(\mathcal{M}, g)$ with metric $d s^{2}=g_{\mu \nu} d x^{\mu} d x^{\nu}$ (with $\mu, \nu=0,1,2,3$ ) and let 
us assume the spacetime has a nonsingular, isolated, compact horizon $\mathcal{H}=\mathcal{H}^{+} \cup \mathcal{H}^{-}$with $\mathcal{H}^{ \pm}=\Sigma_{2} \times \mathbb{R}$, with $\Sigma_{2}$ being a compact spacelike 2-surface, say of topology $S^{2}$. We will consider advanced coordinates $x^{0}=v, x^{A}=z^{A}$ (with $A=1,2$ ) and $x^{3}=\rho$; the horizon is the hypersurface $\rho=0$ on which $v$ is null; $\Sigma_{2}$ is a constant- $v$ section of that hypersurface.

Without loss of generality, we can always choose coordinates such that, close to the future (past) horizon $\mathcal{H}^{+}=\Sigma_{2} \times \mathbb{R}\left(\mathcal{H}^{-}\right)$, the metric takes the form [27,28]

$$
\begin{gathered}
g_{v v}=-2 \kappa \rho+\mathcal{O}\left(\rho^{2}\right), \\
g_{v A}=g_{v A}^{(1)}\left(z^{B}\right) \rho+\mathcal{O}\left(\rho^{2}\right), \\
g_{A B}=g_{A B}^{(0)}\left(z^{C}\right)+g_{A B}^{(1)}\left(z^{C}\right) \rho+\mathcal{O}\left(\rho^{2}\right),
\end{gathered}
$$

together with the gauge fixing conditions

$$
g_{\rho \rho}=0, \quad g_{v \rho}=1, \quad g_{A \rho}=0 .
$$

The radial coordinate $\rho \in \mathbb{R}_{\geq 0}$ measures the distance from the horizon, $v \in \mathbb{R}$ is null on $\mathcal{H}^{+}$(in the case of the past horizon $\mathcal{H}^{-}$the advanced time $v$ has to be replaced by the retarded time $u \in \mathbb{R}$ ), and $z^{A}$ (with $A=1,2$ ) represents coordinates on $\Sigma_{2}$. In (1), $g_{\mu \nu}^{(n)}$ stand for functions of $z^{A}$, each of which can be thought of as the coefficient of the order $\mathcal{O}\left(\rho^{n}\right)$ in the near horizon (i.e., small $\rho$ ) expansion of the metric components. Constant $\kappa=-\frac{1}{2} g_{v v}^{(1)}$ corresponds to the surface gravity of the horizon. We denote $\theta_{A}\left(z^{B}\right) \equiv$ $g_{v A}^{(1)}\left(z^{B}\right)$ and $\Omega_{A B}\left(z^{C}\right) \equiv g_{A B}^{(0)}\left(z^{C}\right)$. The fact that the latter functions are independent of $v$ is guaranteed by the isolated horizon condition.

We are interested in Einstein gravity coupled to YangMills theory, and therefore we have to introduce, in addition to the metric, the non-Abelian gauge field $A_{\mu}^{a}$, which defines the gauge connection 1-form

$$
A=A_{\mu}^{a} T_{a} d x^{\mu},
$$

with $T_{a}$ being the generators of a Lie algebra $\mathrm{g}$ [with $a=1,2, \ldots, \operatorname{dim}(\mathrm{g})]$, which satisfy the Lie product

$$
\left[T_{b}, T_{c}\right]=i f_{b c}^{a} T_{a},
$$

with $f_{b c}^{a}$ being the structure constants. Let $G$ be a compact, semisimple Lie group generated by g, which enters in the definition of the gauge theory through the standard building blocks; we have the covariant derivative $D_{\mu}=$ $\partial_{\mu}-i \alpha A_{\mu}^{a} T_{a}$ and the field strength $F^{a}=F_{\mu \nu}^{a}=\partial_{\mu} A_{\nu}^{a}-$ $\partial_{\nu} A_{\mu}^{a}+\alpha f_{b c}^{a} A_{\mu}^{b} A_{\nu}^{c}$ that yields the g-valued curvature 2-form $F_{\mu \nu}^{a} d x^{\mu} \wedge d x^{\nu} ; \alpha$ is the gauge coupling. The action of the theory reads

$$
I=\frac{1}{16 \pi G} \int_{\mathcal{M}} d^{4} x \sqrt{|g|} R-\frac{1}{4} \int_{\mathcal{M}} d^{4} x \sqrt{|g|} \operatorname{Tr} F^{2}
$$

Close to the horizon, the gauge field satisfies the following asymptotic behavior

$$
\begin{gathered}
A_{v}^{a}=A_{v}^{(0) a}+A_{v}^{(1) a}\left(v, z^{A}\right) \rho+\mathcal{O}\left(\rho^{2}\right), \\
A_{B}^{a}=A_{B}^{(0) a}\left(z^{A}\right)+A_{B}^{(1) a}\left(v, z^{A}\right) \rho+\mathcal{O}\left(\rho^{2}\right),
\end{gathered}
$$

where $A_{\mu}^{(n) a}$ stands for the coefficient of the order $\mathcal{O}\left(\rho^{n}\right)$ in the small- $\rho$ expansion. In addition, the gauge condition $A_{\rho}^{a}=0$ holds for all $\rho$. Notice that, while $A_{\mu}^{(n>0) a}$ are functions of $z^{A}$ and $v, A_{v}^{(0) a}$ is constant, $A_{B}^{(0) a}$ only depends on $z^{A}$; cf. [16]. This suffices to accommodate charged black hole solutions within the configuration space.

Now, having defined the near horizon boundary conditions for both the gravitational and the gauge fields, let us consider the symmetry transformations that preserve such conditions. More precisely, we ask for those diffeomorphisms and gauge transformations that preserve the asymptotic form of (1)-(3) and (8)-(9), allowing for variation of the specific functions $g_{\mu \nu}^{(n)}, A_{\mu}^{(n) a}$ but preserving the functional form of the small $\rho$ expansion together with the gauge-fixing conditions. Let us call $\chi_{\mu}$ and $\epsilon^{a}$ the asymptotic Killing vectors and the gauge parameters that realize such symmetry transformations, respectively. Then, we have the changes $g_{\mu \nu} \rightarrow g_{\mu \nu}+\delta_{\chi} g_{\mu \nu}, A_{\mu}^{a} \rightarrow A_{\mu}^{a}+\delta_{(\chi, \epsilon)} A_{\mu}^{a}$, with

$\delta_{\chi} g_{\mu \nu}=\left(\mathcal{L}_{\chi} g\right)_{\mu \nu}, \quad \delta_{(\chi, \epsilon)} A_{\mu}^{a}=\left(\mathcal{L}_{\chi} A\right)_{\mu}^{a}+\partial_{\mu} \epsilon^{a}-\alpha f_{b c}^{a} \epsilon^{b} A_{\mu}^{c}$.

As said, we require these changes to be such that they preserve the asymptotic conditions prescribed above. For instance, in order to respect the gauge fixing conditions, we have to demand

$$
\begin{array}{ll}
\left(\mathcal{L}_{\chi} g\right)_{\rho \rho}=0, & \left(\mathcal{L}_{\chi} g\right)_{v \rho}=0, \\
\left(\mathcal{L}_{\chi} g\right)_{A \rho}=0, & \left(\mathcal{L}_{\chi} A\right)_{\rho}^{a}+\partial_{\rho} \epsilon^{a}=0,
\end{array}
$$

where we have used $A_{\rho}^{a}=0$. The latter conditions, together with some closure conditions for the asymptotic expansion, yield the following form for $\chi$ and $\epsilon^{a}$

$$
\begin{gathered}
\chi^{v}=f\left(v, z^{A}\right), \\
\chi^{\rho}=-\rho \partial_{v} f+\partial_{A} f \int_{0}^{\rho} g^{A B} g_{v B} d \rho^{\prime}, \\
\chi^{A}=Y^{A}\left(z^{A}\right)-\partial_{B} f \int_{0}^{\rho} g^{A B} d \rho^{\prime},
\end{gathered}
$$


together with

$$
\epsilon^{a}=\epsilon_{0}^{a}\left(v, z^{A}\right)-\int_{0}^{\rho} A_{B}^{a} \partial_{\rho} \chi^{B} d \rho^{\prime} .
$$

Here, $f\left(v, z^{A}\right)$ is a function of $z^{A}$ whose dependence with $v$ will be later restricted by requiring extra conditions. Being the $v$ component of the asymptotic Killing vector, this function will ultimately be associated to horizon supertranslations. Function $\epsilon_{0}^{a}\left(v, z^{A}\right)$ depends on $z^{A}$ and $v$, while $Y^{A}\left(z^{B}\right)$ are two arbitrary functions of $z^{B}$ and do not depend on $v(A, B=1,2)$. On $\Sigma_{2}$ we can consider complex coordinates $\left(z^{1}, z^{2}\right)=(z, \bar{z})$ and holomorphic and antiholomorphic fields obeying $\bar{\partial} Y^{z}(z)=\partial Y^{\bar{z}}(\bar{z})=0$; this allows us to denote $Y=Y^{z}(z) \bar{Y}=Y^{\bar{z}}(\bar{z})$ for short. The latter functions will be identified as those generating local conformal transformations on $\Sigma_{2}$, i.e., they will be related to the so-called supertranslations.

In order to satisfy the boundary conditions (8)-(9), the gauge field has to obey

$$
\begin{aligned}
\left(\mathcal{L}_{\chi} A\right)_{v}^{a}+\partial_{v} \epsilon^{a}-\alpha f_{b c}^{a} \epsilon^{b} A_{v}^{c} & =\mathcal{O}(\rho), \\
\left(\mathcal{L}_{\chi} A\right)_{B}^{a}+\partial_{B} \epsilon^{a}-\alpha f_{b c}^{a} \epsilon^{b} A_{B}^{c} & =\mathcal{O}(1) .
\end{aligned}
$$

Satisfying the first of these conditions implies solving a set of differential equations for $\epsilon_{0}^{a}$; namely

$$
\partial_{v}\left(\chi^{v} A_{v}^{(0) a}+\epsilon_{0}^{a}\right)-\alpha f_{b c}^{a} \epsilon_{0}^{b} A_{v}^{(0) c}=0
$$

where the indices $v$ are not contracted.

By computing the variations of the metric and the gauge field under diffeomorphisms and gauge transformations defined by the $\chi$ and $\epsilon$ given above, one finds

$$
\begin{gathered}
\delta_{(\chi, \epsilon)} \kappa=\kappa \partial_{v} f+\partial_{v}^{2} f=0, \\
\delta_{(\chi, \epsilon)} \theta_{A}=\mathcal{L}_{Y} \theta_{A}+f \partial_{v} \theta_{A}-2 \kappa \partial_{A} f-2 \partial_{v} \partial_{A} f \\
+\Omega^{B C} \partial_{v} \Omega_{A B} \partial_{C} f,
\end{gathered}
$$

$$
\begin{gathered}
\delta_{(\chi, \epsilon)} \Omega_{A B}=f \partial_{v} \Omega_{A B}+\mathcal{L}_{Y} \Omega_{A B}, \\
\delta_{(\chi, \epsilon)} A_{v}^{(0) a}=0, \\
\delta_{(\chi, \epsilon)} A_{B}^{(0) a}=Y^{C} \partial_{C} A_{B}^{(0) a}+A_{C}^{(0) a} \partial_{B} Y^{C}+\partial_{B} f A_{v}^{(0) a} \\
+\partial_{B} \epsilon_{0}^{a}-\alpha f_{b c}^{a} \epsilon_{0}^{b} A_{B}^{(0) c} .
\end{gathered}
$$

Notice that in (18) we are additionally demanding the variation $\delta_{(\chi, \epsilon)} \kappa$ to vanish; that is to say, we are considering a phase space defined by functional variations that preserve the surface gravity. For the case of nonextremal horizons $(\kappa \neq 0)$ this yields $f\left(v, z^{A}\right)=T\left(z^{A}\right)+e^{-\kappa v} X\left(z^{A}\right)$, with $T\left(z^{A}\right)$ and $X\left(z^{A}\right)$ being two arbitrary functions on $\Sigma_{2}$; cf. [10].

\section{SYMMETRY ALGEBRA}

Now, let us derive the algebra that generates the asymptotic symmetries defined above. In order to obtain this algebra, let us start by looking at how two subsequent variations act on the metric function $\theta_{A}$; namely

$$
\begin{aligned}
& {\left[\delta_{\left(\chi_{1}, \epsilon_{1}\right)}, \delta_{\left(\chi_{2}, \epsilon_{2}\right)}\right] \theta_{A}} \\
& \quad=\tilde{Y}^{A} \theta_{A}+\tilde{f} \partial_{v} \theta_{A}-2 \kappa \partial_{A} \tilde{f}-2 \partial_{v} \partial_{A} \tilde{f}+\Omega^{B C} \partial_{v} \Omega_{A B} \partial_{C} \tilde{f},
\end{aligned}
$$

where

$$
\begin{aligned}
\tilde{Y}^{A} & =Y_{1}^{B} \partial_{B} Y_{2}^{A}-Y_{2}^{B} \partial_{B} Y_{1}^{A}, \\
\tilde{f} & =\mathcal{L}_{Y_{1}} f_{2}-\mathcal{L}_{Y_{2}} f_{1}+f_{1} \partial_{v} f_{2}-f_{2} \partial_{v} f_{1} .
\end{aligned}
$$

The brackets [,] in (23) is the modified Lie brackets introduced in [29], which is valid in the case the gauge parameters are field dependent; see Eq. (2.4) therein, see also $[11,26]$ and references thereof.

Now, in order to determine the expression for $\tilde{\epsilon_{0}^{a}}$, it is sufficient to consider the variation of $A_{B}^{(0) a}$ and then see how the algebra closes. This yields the cumbersome expression

$$
\begin{aligned}
{\left[\delta_{\left(\chi_{1}, \epsilon_{1}\right)}, \delta_{\left(\chi_{2}, \epsilon_{2}\right)}\right] A_{B}^{(0) a}=} & \tilde{Y}^{C} \partial_{C} A_{B}^{(0) a}+A_{C}^{(0) a} \partial_{B} \tilde{Y}^{C}+\partial_{B} \tilde{f} A_{v}^{(0) a}+\partial_{B}\left[Y_{1}^{C} \partial_{C} \epsilon_{0_{2}}^{a}-Y_{2}^{C} \partial_{C} \epsilon_{0_{1}}^{a}\right]-\alpha f_{b c}^{a}\left[Y_{1}^{C} \partial_{C} \epsilon_{0_{2}}^{b}-Y_{2}^{C} \partial_{C} \epsilon_{0_{1}}^{b}\right] A_{B}^{(0) c} \\
& -\partial_{B}\left(f_{1} \partial_{v} f_{2}-f_{2} \partial_{v} f_{1}\right) A_{v}^{(0) a}-\alpha f_{b c}^{a}\left[\epsilon_{0_{1}}^{b}\left(\partial_{B} f_{2} A_{v}^{(0) c}+\partial_{B} \epsilon_{0_{2}}^{c}-\alpha f_{j k}^{c} \epsilon_{0_{2}}^{j} A_{B}^{(0) k}\right)\right. \\
& \left.-\epsilon_{0_{2}}^{b}\left(\partial_{B} f_{1} A_{v}^{(0) c}+\partial_{B} \epsilon_{0_{1}}^{c}-\alpha f_{j k}^{c} \epsilon_{0_{1}}^{j} A_{B}^{(0) k}\right)\right]
\end{aligned}
$$

where we have identified some terms, and added and subtracted $\partial_{B}\left(f_{1} \partial_{v} f_{2}-f_{2} \partial_{v} f_{1}\right) A_{v}^{(0) a}$ in order to complete the expression of $\tilde{f}$.

Using Eq. (17) to write $\partial_{v} f A_{v}^{(0) a}=\alpha f_{b c}^{a} \epsilon_{0}^{b} A_{v}^{(0) c}-\partial_{v} \epsilon_{0}^{a}$, the Jacobi identity to write $f_{b c}^{a}\left[f_{m n}^{c}\left(\epsilon_{0_{1}}^{b} \epsilon_{0_{2}}^{m}-\epsilon_{0_{1}}^{m} \epsilon_{0_{2}}^{b}\right) A_{B}^{(0) n}\right]=$ $f_{b c}^{a} f_{m n}^{b} \epsilon_{0_{1}}^{m} \epsilon_{0_{2}}^{n} A_{B}^{(0) c}$, and defining $E^{a} \equiv Y_{1}^{C} \partial_{C} \epsilon_{0_{2}}^{a}-Y_{2}^{C} \partial_{C} \epsilon_{0_{1}}^{a}+f_{1} \partial_{v} \epsilon_{0_{2}}^{a}-f_{2} \partial_{v} \epsilon_{0_{1}}^{a}$ for short, the expression above can be rewritten as follows: 


$$
\begin{aligned}
{\left[\delta_{\left(\chi_{1}, \epsilon_{1}\right)}, \delta_{\left(\chi_{2}, \epsilon_{2}\right)}\right] A_{B}^{(0) a}=} & \tilde{Y}^{C} \partial_{C} A_{B}^{(0) a}+A_{C}^{(0) a} \partial_{B} \tilde{Y}^{C}+\partial_{B} \tilde{f} A_{v}^{(0) a}+\partial_{B}\left(E^{a}-\alpha f_{b c}^{a} \epsilon_{0_{1}}^{b} \epsilon_{0_{2}}^{c}\right)-\alpha f_{b c}^{a}\left(E^{b}-\alpha f_{m n}^{b} \epsilon_{0_{1}}^{m} \epsilon_{0_{2}}^{n}\right) A_{B}^{(0) c} \\
& +f_{1} \partial_{v}\left[\partial_{B}\left(\epsilon_{0_{2}}^{a}+f_{2} A_{v}^{(0) a}\right)-\alpha f_{b c}^{a} \epsilon_{0_{2}}^{b} A_{B}^{(0) c}\right]-f_{2} \partial_{v}\left[\partial_{B}\left(\epsilon_{0_{1}}^{a}+f_{1} A_{v}^{(0) a}\right)-\alpha f_{b c}^{a} \epsilon_{0_{1}}^{b} A_{B}^{(0) c}\right] .
\end{aligned}
$$

We are interested in writing this expression as

$$
\begin{aligned}
\delta_{(\tilde{\chi}, \tilde{\epsilon})} A_{B}^{(0) a}= & \tilde{Y}^{C} \partial_{C} A_{B}^{(0) a}+A_{C}^{(0) a} \partial_{B} \tilde{Y}^{C}+\partial_{B} \tilde{f} A_{v}^{(0) a} \\
& +\partial_{B} \tilde{\epsilon_{0}^{a}}-\alpha f_{b c}^{a} \tilde{\epsilon}_{0}^{b} A_{B}^{(0) c}
\end{aligned}
$$

for a given $\tilde{\epsilon_{0}^{b}}$. Using that $A_{B}^{(0) a}$ and $Y^{A}$ only depend on $z^{A}$, we can freely add the term $\partial_{v}\left[Y^{C} \partial_{C} A_{B}^{(0) a}+A_{C}^{(0) a} \partial_{B} Y^{C}\right]$ to (26) and then identify the last line of that equation as $f_{1} \partial_{v}\left(\delta_{\left(\tilde{\chi_{2}}, \tilde{\varepsilon_{2}}\right)} A_{B}^{(0) a}\right)-f_{2} \partial_{v}\left(\delta_{\left(\tilde{\chi_{1}}, \tilde{\epsilon_{1}}\right)} A_{B}^{(0) a}\right)$, which vanishes as $\delta_{(\tilde{\chi}, \tilde{\epsilon})} A_{B}^{(0) a}$ only depends on $z^{A}$. This allows us to identify $\tilde{\epsilon_{0}^{a}}=E^{a}-\alpha f_{b c}^{a} \epsilon_{0_{1}}^{b} \epsilon_{0_{2}}^{c}$, which reduces to the expression of [16] in the Abelian case $f_{c}^{a b}=0$. In the most general case, we find the following set of variations

$$
\begin{gathered}
\tilde{Y}^{A}=Y_{1}^{B} \partial_{B} Y_{2}^{A}-Y_{2}^{B} \partial_{B} Y_{1}^{A}, \\
\tilde{f}=Y_{1}^{C} \partial_{C} f_{2}-Y_{2}^{C} \partial_{C} f_{1}+f_{1} \partial_{v} f_{2}-f_{2} \partial_{v} f_{1},
\end{gathered}
$$

$\tilde{\epsilon_{0}^{a}}=Y_{1}^{C} \partial_{C} \epsilon_{0_{2}}^{a}-Y_{2}^{C} \partial_{C} \epsilon_{0_{1}}^{a}+f_{1} \partial_{v} \epsilon_{0_{2}}^{a}-f_{2} \partial_{v} \epsilon_{0_{1}}^{a}-\alpha f_{b c}^{a} \epsilon_{0_{1}}^{b} \epsilon_{0_{2}}^{c}$.

Now, we can solve Eq. (17) and replace its solution in (30). Equation (17) can be written as $\partial_{v}\left(\epsilon_{0}^{a}+f A_{v}^{(0) a}\right)=$ $\Delta_{b}^{a} \epsilon_{0}^{b}$ with $\Delta_{b}^{a}=\alpha f_{b c}^{a} A_{v}^{(0) c}$, which has solution of the form

$$
\epsilon_{0}^{a}\left(v, z^{A}\right)=\left[e^{\Delta v}\right]_{b}^{a} U^{b}\left(z^{A}\right)-f\left(z^{A}, v\right) A_{v}^{(0) a} .
$$

This generalizes the result $\epsilon_{0}^{a}\left(v, z^{A}\right)=U^{a}\left(z^{A}\right)-$ $f\left(z^{A}, v\right) A_{v}^{(0) a}$ with $a=1,2, \ldots N$ obtained in [16] for $G=U(1)^{N}$. The novel feature in (31) relative to the Abelian case is the $v$-dependent exponential accompanying the function $U^{a}\left(z^{A}\right)$, which obviously vanishes when the structure constants vanish.

Having fully determined the functional dependence with the advanced null coordinate $v$, it is possible to obtain the expression for $\tilde{U}^{a}$ explicitly; namely

$$
\begin{aligned}
\tilde{\epsilon_{0}^{a}}+\tilde{f} A_{v}^{(0) a}= & {\left[e^{\Delta v}\right]_{j}^{a} \tilde{U}^{j}\left(z^{A}\right) } \\
= & {\left[e^{\Delta v}\right]_{j}^{a}\left[Y_{1}^{C} \partial_{C} U_{2}^{j}-Y_{2}^{C} \partial_{C} U_{1}^{j}\right] } \\
& -\alpha f_{b c}^{a}\left[e^{\Delta v}\right]_{k}^{b}\left[e^{\Delta v}\right]_{m}^{c} U_{1}^{k} U_{2}^{m},
\end{aligned}
$$

where we see that the dependence of $f_{1}$ and $f_{2}$ has cancelled out. We can use that $f_{b c}^{a}\left[e^{\Delta v}\right]_{k}^{b}\left[e^{\Delta v}\right]_{m}^{c}=f_{k m}^{j}\left[e^{\Delta v}\right]_{j}^{a}$, which follows from standard formulas applied to the adjoint representation. Taking all this into account, one finally obtains

$$
\tilde{U}^{a}=Y_{1}^{C} \partial_{C} U_{2}^{a}-Y_{2}^{C} \partial_{C} U_{1}^{a}-\alpha f_{b c}^{a} U_{1}^{b} U_{2}^{c}
$$

which, together with the transformations

$$
\begin{gathered}
\tilde{Y}^{A}=Y_{1}^{B} \partial_{B} Y_{2}^{A}-Y_{2}^{B} \partial_{B} Y_{1}^{A}, \\
\tilde{T}=Y_{1}^{B} \partial_{B} T_{2}-Y_{2}^{B} \partial_{B} T_{1}, \\
\tilde{X}=Y_{1}^{B} \partial_{B} X_{2}-\kappa T_{1} X_{2}-Y_{2}^{B} \partial_{B} X_{1}+\kappa T_{2} X_{1},
\end{gathered}
$$

form the symmetry algebra. Functions $Y^{B}, T, X$, and $U^{a}$ are four arbitrary functions of the coordinates $z^{A}$ on $\Sigma_{2}$, and this gives rise to an infinite-dimensional algebra. While the line (34) expresses the existence of two copies of the Witt algebra (namely; two Virasoro algebras with a vanishing central term), the line (35) gives the semidirect sum of the Virasoro algebras with an Abelian infinite-dimensional algebra i.e., the so-called supertranslations. The line (36) shows the presence of another infinite-dimensional Abelian component, which acts on supertranslations as dilations act on standard translations. The new result here is (33). This expresses, on the one hand, the mixing between the spacetime superrotations and the gauge transformations at the horizon; on the other hand, it generalizes the result of [16] to the non-Abelian case, where the $G$-structure appears in the last term with the structure constants.

All the arbitrary functions of $z^{A}$ can be expanded in Fourier modes, e.g., as usually done when representing $\operatorname{Diff}\left(S^{1}\right)$ or tensored $C^{\infty}\left(S^{1}\right)$ algebras in conformal field theory. It amounts to choose complex coordinates $z^{A}=(z, \bar{z})$ with $z=e^{\tau+i \sigma}$, and evaluate (33)-(34) on arbitrary modes $z^{m} \bar{z}^{n}$. That is to say, we can define $T(z, \bar{z})=\kappa \sum_{m, n} T_{(m, n)} z^{m} \bar{z}^{n}, \quad X(z, \bar{z})=\sum_{m, n} X_{(m, n)} z^{m} \bar{z}^{n}$, $Y(z)=\sum_{n} Y_{n} z^{n}, \quad \bar{Y}(\bar{z})=\sum_{n} \bar{Y}_{n} \bar{z}^{n} \quad$ and $\quad U^{a}(z, \bar{z})=$ $\alpha \sum_{m, n} U_{(m, n)}^{a} z^{m} \bar{z}^{n}$, and then express the algebra as follows:

$$
\begin{aligned}
& {\left[Y_{m}, Y_{n}\right]=(m-n) Y_{m+n}, \quad\left[\bar{Y}_{m}, \bar{Y}_{n}\right]=(m-n) \bar{Y}_{m+n},} \\
& {\left[Y_{k}, T_{(m, n)}\right]=-m T_{(m+k, n)}, \quad\left[\bar{Y}_{k}, T_{(m, n)}\right]=-n T_{(m, n+k)},} \\
& {\left[Y_{k}, X_{(m, n)}\right]=-m X_{(m+k, n)}, \quad\left[\bar{Y}_{k}, X_{(m, n)}\right]=-n X_{(m, n+k)},}
\end{aligned}
$$




$$
\left[Y_{k}, U_{(m, n)}^{a}\right]=-m U_{(m+k, n)}^{a}, \quad\left[\bar{Y}_{k}, U_{(m, n)}^{a}\right]=-n U_{(m, n+k)}^{a},
$$

$$
\begin{aligned}
{\left[X_{(k, l)}, T_{(m, n)}\right] } & =X_{(m+k, n+l)}, \\
{\left[U_{(k, l)}^{a}, U_{(m, n)}^{b}\right] } & =f_{c}^{a b} U_{(k+m, l+n)}^{c} ;
\end{aligned}
$$

the other commutators vanish.

The last Lie product in (41) is our main result. This manifestly shows that in the near horizon region of black holes in Einstein-Yang-Mills theory the symmetry algebra gets enhanced in such a way that it includes a (double) infinite-dimensional loop algebra $\hat{\mathrm{g}}=\mathrm{g} \otimes C^{\infty}\left(S^{1}\right)$ generated by $U_{(m, n)}^{a}$. This algebra is in semidirect sum with two copies of Witt algebra, being a spin-1 current under local conformal transformations. This generalizes the result found in [16] for the $U(1)^{N}$ theory, which here corresponds to $f_{b c}^{a}=0$. The rest of the algebra matches the one found in $[10,11]$; namely, in addition to the loop algebra, the algebra above contains an infinite-dimensional Abelian piece generated by $T_{(m, n)}$, which is the horizon supertranslations. There is also an infinite-dimensional Abelian ideal generated by $X_{(m, n)}$. However, as shown in $[10,11]$, in the case of nonextremal black holes the latter algebra generates transformations that are pure gauge as $X(z, \bar{z})$ does not enter in the Noether charges associated to the asymptotic diffeomorphisms generated by $\chi=f(v, z, \bar{z}) \partial_{v}$. We intend to return to the problem of computing the Noether charges associated to the full algebra (37)-(41) and carefully analyzing their integrability conditions in a future work. Preliminary results on this permits to say that the conserved charges associated to $Y_{m}, \bar{Y}_{m}$, and $U_{(m, n)}^{a}$ form a Virasoro-Kac-Moody system with vanishing central charge and vanishing Kac-Moody level.

\section{DISCUSSION}

The results obtained here might have interesting applications to study colored black holes [30,31], cf. [32]. In fact, it is possible to speculate that large gauge transformations could describe physical processes that produce a splash of colors on the horizon, for instance by means of a mechanism similar to the one studied in $[12,16]$. As in there, one can imagine dynamical processes that connect the asymptotic past null-infinity $\mathcal{I}^{-}$with the future horizon $\mathcal{H}^{+}$, using that now we have learnt that a similar Virasoro-Kac-Moody structure emerges in both regions $[26,33,34]$.

Besides, the formulas above can easily be extended to higher dimensions [35], and so applied to study selfgravitating Yang monopoles in arbitrary (even) dimension $D$. Such monopoles have been constructed in [36] for the gauge group $S O(D-2)$. For $D \geq 6$, such solutions describe non-Abelian black holes that, when the cosmological constant is negative, can have applications within the context of AdS/CFT correspondence, cf. [36]. It is possible to show that the analysis done in the previous sections applies to those non-Abelian solutions, and that the zero-mode of the Noether charges associated to the horizon supertranslations correctly reproduces the entropy of the black holes, cf. [37]. This is quite interesting since the selfgravitating Yang monopole has infinite gravitational energy and, therefore, the charge computation needed to study, for example, its thermodynamics only makes sense from the horizon perspective.

Other interesting questions related to the computation presented here relate to its possible generalizations. For example, one could ask whether a more general set of asymptotic conditions exist yielding non-vanishing central extensions of the charge algebra. We could also ask whether other algebraic structures, such as $w_{N}$ algebras, or even $w_{\infty+1}$ algebras as the recently found at null infinity [38], can also be realized at the horizon provided one prescribes adequate asymptotic conditions. These are all interesting questions for future investigations.

\section{ACKNOWLEDGMENTS}

The authors are indebted to Mauricio Leston for discussions. This work is based on previous collaborations with Laura Donnay and Hernán González. G. G. thanks them for many enjoyable and illuminating conversations. This work has been partially supported by CONICET and ANPCyT through Grants No. PIP-1109-2017 and No. PICT-2019-00303.
[1] S. W. Hawking, The information paradox for black holes, arXiv:1509.01147.

[2] H. Bondi, M. G. J. van der Burg, and A. W. K. Metzner, Gravitational waves in general relativity. 7. Waves from axisymmetric isolated systems, Proc. R. Soc. A 269, 21 (1962).
[3] R. K. Sachs, Gravitational waves in general relativity. 8. Waves in asymptotically flat space-times, Proc. R. Soc. A 270, 103 (1962).

[4] R. Sachs, Asymptotic symmetries in gravitational theory, Phys. Rev. 128, 2851 (1962). 
[5] A. Strominger, Lectures on the infrared structure of gravity and gauge theory, arXiv:1703.05448.

[6] S. W. Hawking, M. J. Perry, and A. Strominger, Soft Hair on Black Holes, Phys. Rev. Lett. 116, 231301 (2016).

[7] M. Mirbabayi and M. Porrati, Dressed Hard States and Black Hole Soft Hair, Phys. Rev. Lett. 117, 211301 (2016).

[8] R. Bousso and M. Porrati, Soft hair as a soft wig, Classical Quantum Gravity 34, 204001 (2017).

[9] R. Bousso and M. Porrati, Observable supertranslations, Phys. Rev. D 96, 086016 (2017).

[10] L. Donnay, G. Giribet, H. A. Gonzalez, and M. Pino, Supertranslations and Superrotations at the Black Hole Horizon, Phys. Rev. Lett. 116, 091101 (2016).

[11] L. Donnay, G. Giribet, H. A. González, and M. Pino, Extended symmetries at the black hole horizon, J. High Energy Phys. 09 (2016) 100.

[12] S. W. Hawking, M. J. Perry, and A. Strominger, Superrotation charge and supertranslation hair on black holes, J. High Energy Phys. 05 (2017) 161.

[13] J. I. Koga, Asymptotic symmetries on Killing horizons, Phys. Rev. D 64, 124012 (2001).

[14] M. Hotta, K. Sasaki, and T. Sasaki, Diffeomorphism on horizon as an asymptotic isometry of Schwarzschild black hole, Classical Quantum Gravity 18, 1823 (2001).

[15] M. Hotta, Holographic charge excitations on horizontal boundary, Phys. Rev. D 66, 124021 (2002).

[16] L. Donnay, G. Giribet, H. A. González, and A. Puhm, Black hole memory effect, Phys. Rev. D 98, 124016 (2018).

[17] L. Donnay and C. Marteau, Carrollian physics at the black hole horizon, Classical Quantum Gravity 36, 165002 (2019).

[18] D. Grumiller, A. Pérez, M. M. Sheikh-Jabbari, R. Troncoso, and C. Zwikel, Spacetime Structure Near Generic Horizons and Soft Hair, Phys. Rev. Lett. 124, 041601 (2020).

[19] H. Afshar, D. Grumiller, and M. M. Sheikh-Jabbari, Near horizon soft hair as microstates of three dimensional black holes, Phys. Rev. D 96, 084032 (2017).

[20] R. F. Penna, Near-horizon BMS symmetries as fluid symmetries, J. High Energy Phys. 10 (2017) 049.

[21] P. Mao, X. Wu, and H. Zhang, Soft hairs on isolated horizon implanted by electromagnetic fields, Classical Quantum Gravity 34, 055003 (2017).

[22] A. A. Rahman and R. M. Wald, Black hole memory, Phys. Rev. D 101, 124010 (2020).
[23] M. Blau and M. O'Loughlin, Horizon shells and BMS-like soldering transformations, J. High Energy Phys. 03 (2016) 029.

[24] S. Carlip, Black Hole Entropy from Bondi-Metzner-Sachs Symmetry at the Horizon, Phys. Rev. Lett. 120, 101301 (2018).

[25] R. G. Cai, S. M. Ruan, and Y. L. Zhang, Horizon supertranslation and degenerate black hole solutions, J. High Energy Phys. 09 (2016) 163.

[26] G. Barnich and P. H. Lambert, Einstein-Yang-Mills theory: Asymptotic symmetries, Phys. Rev. D 88, 103006 (2013).

[27] I. Booth, Spacetime near isolated and dynamical trapping horizons, Phys. Rev. D 87, 024008 (2013).

[28] V. Moncrief and J. Isenberg, Symmetries of cosmological Cauchy horizons with non-closed orbits, Commun. Math. Phys. 374, 145 (2020).

[29] G. Barnich and C. Troessaert, Aspects of the BMS/CFT correspondence, J. High Energy Phys. 05 (2010) 062.

[30] P. Bizon, Colored Black Holes, Phys. Rev. Lett. 64, 2844 (1990).

[31] M. S. Volkov and D. V. Gal'tsov, Gravitating nonAbelian solitons and black holes with Yang-Mills fields, Phys. Rep. 319, 1 (1999).

[32] R. Bartnik and J. Mckinnon, Particle-Like Solutions of the Einstein Yang-Mills Equations, Phys. Rev. Lett. 61, 141 (1988).

[33] M. Campiglia and J. Peraza, Charge algebra for non-Abelian large gauge symmetries at $O(r)$, J. High Energy Phys. 12 (2021) 058.

[34] N. Banerjee, T. Rahnuma, and R. K. Singh, Asymptotic symmetry of four dimensional Einstein-Yang-Mills and Einstein-Maxwell theory, J. High Energy Phys. 01 (2022) 033.

[35] C. Shi and J. Mei, Extended symmetries at black hole horizons in generic dimensions, Phys. Rev. D 95, 104053 (2017).

[36] G. W. Gibbons and P. K. Townsend, Self-gravitating Yang monopoles in all dimensions, Classical Quantum Gravity 23, 4873 (2006).

[37] R. M. Wald, Black hole entropy is the Noether charge, Phys. Rev. D 48, R3427 (1993).

[38] A. Strominger, $w(1+$ infinity $)$ and the celestial sphere, arXiv:2105.14346. 